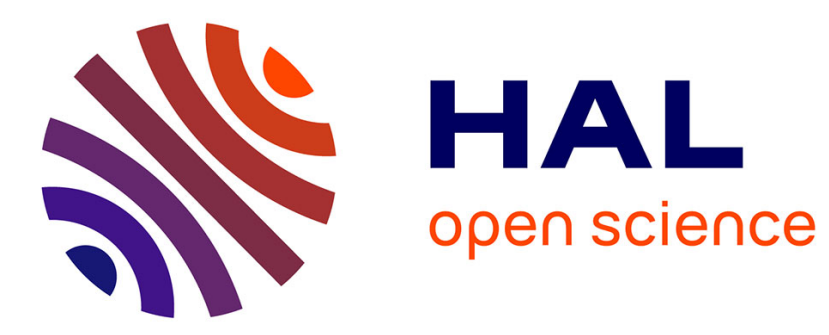

\title{
Asymptotic analysis of a thin interface: the case involving similar rigidity
}

Frédéric Lebon, Raffaella Rizzoni

\section{To cite this version:}

Frédéric Lebon, Raffaella Rizzoni. Asymptotic analysis of a thin interface: the case involving similar rigidity. International Journal of Engineering Science, 2010, 48 (5), pp.473-486. 10.1016/j.ijengsci.2009.12.001 . hal-00459507

\section{HAL Id: hal-00459507 https://hal.science/hal-00459507}

Submitted on 19 Jun 2018

HAL is a multi-disciplinary open access archive for the deposit and dissemination of scientific research documents, whether they are published or not. The documents may come from teaching and research institutions in France or abroad, or from public or private research centers.
L'archive ouverte pluridisciplinaire HAL, est destinée au dépôt et à la diffusion de documents scientifiques de niveau recherche, publiés ou non, émanant des établissements d'enseignement et de recherche français ou étrangers, des laboratoires publics ou privés. 


\title{
Asymptotic analysis of a thin interface: The case involving similar rigidity
}

\author{
F. Lebon ${ }^{\mathrm{a}, *}$, R. Rizzoni $^{\mathrm{b}}$ \\ ${ }^{a}$ Laboratoire de Mécanique et d'Acoustique, Aix-Marseille 1 University-CNRS, 31, Chemin Joseph Aiguier, 13402 Marseille Cedex 20, France \\ ${ }^{\mathrm{b}}$ Dipartimento di Ingegneria, Universitá di Ferrara, Ferrara, Italy
}

\begin{abstract}
This study deals with a linear elastic body consisting of two solids connected by a thin adhesive interphase with a small thickness $\varepsilon$. The three parts have similar elastic moduli. It is proposed to model the limit behavior of the interphase when $\varepsilon \rightarrow 0$. It has been established [1], using matched asymptotic expansions, that at order zero, the interphase reduces to a perfect interface, while at order one, the interphase behaves like an imperfect interface, with a transmission condition involving the displacement and the traction vectors at order zero. The perfect interface model is exactly recovered using a $\Gamma$-convergence argument. At a higher order, a new model of imperfect interface is obtained by studying the properties of a suitable (weakly converging) sequence of equilibrium solutions. Some analytical examples are given to illustrate the results obtained.
\end{abstract}

\section{Introduction}

Interphases play a crucial role in the analysis of structure assemblies, especially in glue-bonding processes [27]. However, due to their small thickness (typically in the $1 \mu \mathrm{m}$ to $1 \mathrm{~mm}$ range), it is difficult to account directly for these interfaces in a complete finite element analysis of a structure. One possible strategy consists in undertaking an asymptotic analysis (where the small parameter is the thickness $\varepsilon$ of the interphase) to eliminate the interphase geometrically and to obtain an equivalent interface model which will be simpler to implement in numerical simulations. This idea has been used in many studies to obtain interface laws linking the stress vector to the jump in the displacement vector at the interface [2,3,5,7,13-15,19$21,23-25,28-30,32,35,36]$, similar to the phenomenological laws described in the literature [16-18]. In many of these studies, a soft interphase, has often been assumed to exist, with a much lower stiffness than that of the adherents (the stiffness is another small parameter). Fewer studies have focused on joints consisting of adherents and an interphase with a comparable level of rigidity [1,8,26,31]. It has been established [1], using matched asymptotic expansions [12,33], that at the first order $(\varepsilon \rightarrow 0)$ one obtains a perfect interface model, which prescribes the vanishing of the jumps in the stress and the displacement vectors. At an higher order (the second term in the expansion), an imperfect interface model is obtained, with a transmission condition involving the first order displacement and traction vectors and their derivatives. The presence of these derivatives reflects non-locality.

The aim of this study was to conduct a rigorous asymptotic analysis via variational convergence $(\Gamma$-convergence $[6,9,11])$ of the equilibrium problem in the case of a body comprising interphase and adherents with comparable rigidities. The results obtained are compared with those presented in [1] and the imperfect interface law is illustrated by presenting some simple examples.

This paper is organized as follows: In Section 2, the mechanical problem is presented. In Section 3, the problem is analyzed at order zero, using $\Gamma$-convergence theory. In Section 4, an example of the results obtained in Section 3 is given. In

\footnotetext{
* Corresponding author.

E-mail address: lebon@lma.cnrs-mrs.fr (F. Lebon).
} 
Section 5, the asymptotic study is performed at a higher order and the results are presented in Section 6. The proof of these results is given in Section 7.

\section{The mechanical problem}

We consider a body occupying an open bounded set $\Omega$ of $\mathbb{R}^{3}$, with a smooth boundary $\partial \Omega$. Let $\left(O, e_{1}, e_{2}, e_{3}\right)$ be an orthonormal frame in the three-dimensional space. The set $\Omega$ is assumed to have a non-empty intersection $S$ with the plane $\left\{x_{3}=0\right\}$. Introducing a small parameter $\varepsilon>0$, we define the following domains:

$$
\begin{aligned}
& B^{\varepsilon}=\left\{\left(x_{1}, x_{2}, x_{3}\right) \in \Omega:\left|x_{3}\right|<\frac{\varepsilon}{2}\right\}, \\
& \Omega^{\varepsilon}=\left\{\left(x_{1}, x_{2}, x_{3}\right) \in \Omega:\left|x_{3}\right|>\frac{\varepsilon}{2}\right\}, \\
& \Omega_{ \pm}^{\varepsilon}=\left\{\left(x_{1}, x_{2}, x_{3}\right) \in \Omega: \pm x_{3}>\frac{\varepsilon}{2}\right\}, \\
& S_{ \pm}^{\varepsilon}=\left\{\left(x_{1}, x_{2}, x_{3}\right) \in \Omega: \pm x_{3}=\frac{\varepsilon}{2}\right\}, \\
& \Omega_{ \pm}=\left\{\left(x_{1}, x_{2}, x_{3}\right) \in \Omega: \pm x_{3}>0\right\}, \\
& S=\left\{\left(x_{1}, x_{2}, x_{3}\right) \in \Omega: x_{3}=0\right\}, \\
& \Omega_{0}=\Omega_{+} \cup \Omega_{-} .
\end{aligned}
$$

The sets $B^{\varepsilon}$ and $\Omega^{\varepsilon}$ are the domains occupied by the adhesive and the adherents, respectively, the set $\Omega^{0}$ is the geometrical limit of $\Omega^{\varepsilon}, S_{ \pm}^{\varepsilon}$ are the interfaces between the adhesive and the adherents and $S$ is the surface to which the adhesive tends geometrically (Fig. 1).

The structure is subjected to a body force density $\varphi$ and a surface force density $g$ on part $\Gamma_{1}$ of the boundary, whereas it is clamped on the remaining part $\Gamma_{0}$ of the boundary. We take $\sigma^{\varepsilon}$ to denote the stress tensor and $u^{\varepsilon}$ to denote the displacement field. In the infinitesimal case, the strain tensor is the symmetric part of the displacement gradient

$$
e_{i j}\left(u^{\varepsilon}\right)=\frac{1}{2}\left(\frac{\partial u_{i}^{\varepsilon}}{\partial x_{j}}+\frac{\partial u_{j}^{\varepsilon}}{\partial x_{i}}\right)
$$

The adherents and the adhesive are assumed to be linearly elastic and $a_{i j k l}^{ \pm}$is taken to denote the elasticity coefficients of the adherents and $a_{i j k l}^{m}$ to denote the elasticity coefficients of the adhesive.

For a given function $f: \Omega \mapsto R^{3}$, we define the restrictions of $f$ to the adherents by $f_{\varepsilon}^{ \pm}$and to the adhesive by $f_{\varepsilon}^{m}$. Taking $\hat{x}=\left(x_{1}, x_{2}\right)$ to denote the in-plane coordinates of the adhesive, we define the following jumps of $f$

$$
\begin{aligned}
& {[f]_{\varepsilon}^{+}(\hat{x}):=f_{\varepsilon}^{+}\left(x_{1}, x_{2},\left(\frac{\varepsilon}{2}\right)^{+}\right)-f_{\varepsilon}^{m}\left(x_{1}, x_{2},\left(\frac{\varepsilon}{2}\right)^{-}\right),} \\
& {[f]_{\varepsilon}^{-}(\hat{x}):=f_{\varepsilon}^{-}\left(x_{1}, x_{2},\left(-\frac{\varepsilon}{2}\right)^{-}\right)-f_{\varepsilon}^{m}\left(x_{1}, x_{2},\left(-\frac{\varepsilon}{2}\right)^{+}\right),} \\
& {[f]_{\varepsilon}(\hat{x}):=f_{\varepsilon}^{m}\left(x_{1}, x_{2},\left(\frac{\varepsilon}{2}\right)^{-}\right)-f_{\varepsilon}^{m}\left(x_{1}, x_{2},\left(-\frac{\varepsilon}{2}\right)^{+}\right) .}
\end{aligned}
$$

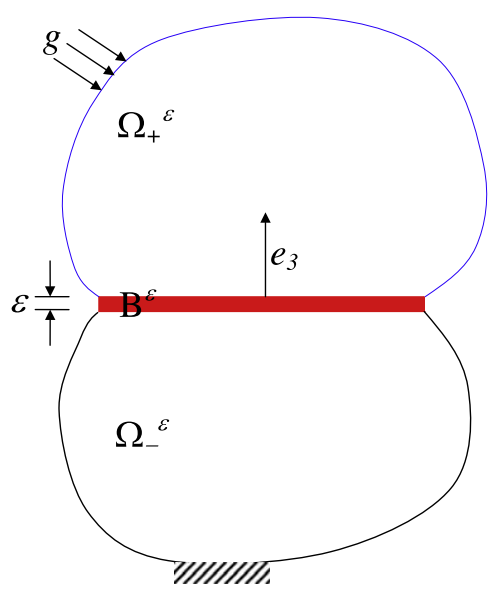

(a)

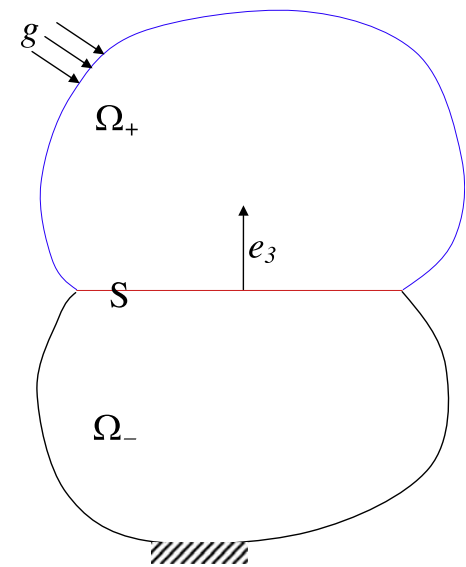

(b)

Fig. 1. (a) Reference and (b) limit configurations of the joint. 
For a given function $f: \Omega_{0} \mapsto R^{3}$, we define the restrictions of $f$ to $\Omega_{ \pm}$by $f^{ \pm}$and we also define the following jump of $f$ on $S$

$$
[f](\hat{x}):=f^{+}\left(x_{1}, x_{2}, 0^{+}\right)-f^{-}\left(x_{1}, x_{2}, 0^{-}\right) .
$$

Writing the local expression for the equilibrium problem corresponds to finding the displacement $u^{\varepsilon}$, strain $e\left(u^{\varepsilon}\right)$ and stress $\sigma^{\varepsilon}$ fields which solve the following problem:

$$
\left(P_{\varepsilon}\right)\left\{\begin{array}{l}
\text { Find }\left(u^{\varepsilon}, \sigma^{\varepsilon}\right) \text { such that : } \\
\sigma_{i j, j}^{\varepsilon}=-\varphi_{i} \quad \text { in } \Omega, \\
\sigma_{i j}^{\varepsilon}=a_{i j k h}^{ \pm} e_{k h}\left(u^{\varepsilon}\right) \quad \text { in } \Omega_{ \pm}^{\varepsilon}, \\
\sigma_{i j}^{\varepsilon}=a_{i j k h}^{m} e_{k h}\left(u^{\varepsilon}\right) \quad \text { in } B^{\varepsilon} \\
u^{\varepsilon}=0 \quad \text { on } \Gamma_{0}, \\
\sigma_{\varepsilon} n=g \quad \text { on } \Gamma_{1} \\
{\left[u^{\varepsilon}\right]_{\varepsilon}^{ \pm}=0, \quad\left[\sigma^{\varepsilon} e_{3}\right]_{\varepsilon}^{ \pm}=0 .}
\end{array}\right.
$$

We now introduce the space of kinematically admissible displacements

$$
V^{\varepsilon}=\left\{u \in\left(W^{1,2}(\Omega)\right)^{3}: u=0 \text { on } \Gamma_{0}\right\},
$$

and the work of the internal and external loads, respectively,

$$
\begin{aligned}
& A^{\varepsilon}(u, v)=\int_{\Omega_{+}^{\varepsilon}} a^{+} e(u): e(v) d x+\int_{\Omega_{-}^{\varepsilon}} a^{-} e(u): e(v) d x+\int_{B^{\varepsilon}} a^{m} e(u): e(v) d x, \\
& l^{\varepsilon}(v)=\int_{\Omega} \phi \cdot v d x+\int_{\Gamma_{1}} g \cdot v d s .
\end{aligned}
$$

Under the following regularity assumptions

$$
\begin{aligned}
& (\text { H1 }) \quad\left\{\begin{array}{l}
a_{i j k l} \in L^{\infty}(\Omega), \\
a_{i j k l}=a_{k l i j}=a_{j i k}, \\
\exists \eta>0: a_{i j k l} e_{i j} e_{k l} \geqslant \eta e_{i j} e_{i j} \quad \forall e_{i j}=e_{j i},
\end{array}\right. \\
& \left(\text { H2) } \exists \varepsilon_{0}: B_{\varepsilon} \cap\left(\Gamma_{1} \cup \operatorname{supp}(\phi)\right)=\emptyset, \quad \forall \varepsilon<\varepsilon_{0},\right.
\end{aligned}
$$

we can reformulate $\left(P_{\varepsilon}\right)$ as follows:

$$
\left(\bar{P}_{\varepsilon}\right) \quad\left\{\begin{array}{l}
\text { Find } u^{\varepsilon} \in V^{\varepsilon}: \\
A\left(u^{\varepsilon}, v\right)=l^{\varepsilon}(v) \quad \forall v \in V^{\varepsilon} .
\end{array}\right.
$$

In view of the Lax-Milgram Lemma, this problems has a unique solution, which is also the unique solution of the following minimum problem:

$$
\left(\widetilde{P}_{\varepsilon}\right) \quad\left\{\begin{array}{l}
\text { Find } u^{\varepsilon} \in V^{\varepsilon}: \\
J^{\varepsilon}\left(u^{\varepsilon}\right) \leqslant J^{\varepsilon}(v) \quad \forall v \in V^{\varepsilon}
\end{array}\right.
$$

where

$$
J^{\varepsilon}(v)=\frac{1}{2} A^{\varepsilon}(v, v)-l^{\varepsilon}(v)
$$

is the potential energy associated with the displacement field $v$. We define the functional spaces

$$
\begin{aligned}
& V^{\varepsilon}=\left\{u \in\left(W^{1,2}(\Omega)\right)^{3}: u=0 \text { on } \Gamma_{0}\right\}, \\
& X=\left(L^{2}(\Omega)\right)^{3} .
\end{aligned}
$$

Finally, we introduce the strain energy functional

$$
F^{\varepsilon}(v)=\left\{\begin{array}{l}
\frac{1}{2} A^{\varepsilon}(v, v) \text { if } v \in V^{\varepsilon} \\
+\infty \text { if } v \in X \backslash V^{\varepsilon}
\end{array}\right.
$$

\section{Zero order results}

We introduce the limit functional 


$$
F^{0}(v)=\left\{\begin{array}{l}
\int_{\Omega_{0}} a^{ \pm} e(v): e(v) d x \quad \text { if } v \in V^{0}, \\
+\infty \quad \text { if } v \in X \backslash V^{0}
\end{array}\right.
$$

where

$$
V^{0}=\left\{u \in\left(W^{1,2}\left(\Omega_{0}\right)\right)^{3}: u=0 \text { on } \Gamma_{0},[v]=0 \text { on } S\right\} .
$$

Theorem 1. The sequence of functionals $F^{\varepsilon} \Gamma$-converges for the strong topology of $X$ to $F^{0}$.

To prove Theorem 1, we need the two following Lemmas, in which we take $C$ to denote any constant independent of $\varepsilon$. The first Lemma is that obtained by Licht and Michaille [30]. Lemma 2 is proved in Appendix 1.

Lemma 1. For all $v \in V^{\varepsilon}$, there exists a constant $C>0$ independent of $\varepsilon$ such that

$$
\begin{aligned}
& \int_{B^{\varepsilon}}|v(x)|^{2} d x \leqslant C\left(\varepsilon^{2} \int_{B^{\varepsilon}}|e(v(x))|^{2} d x+\varepsilon \int_{\Omega^{\varepsilon}}|e(v(x))|^{2} d x\right), \\
& \int_{\Omega^{\varepsilon}}|v(x)|^{2} d x \leqslant C \int_{\Omega^{\varepsilon}}|e(v(x))|^{2} d x, \\
& \int_{\Gamma_{1}}|v(x)|^{2} d x \leqslant C \int_{\Omega^{\varepsilon}}|e(v(x))|^{2} d x .
\end{aligned}
$$

Lemma 2. There exist constants $C>0$ such that

$$
\begin{aligned}
& \int_{\Omega}\left|u^{\varepsilon}(x)\right|^{2} d x \leqslant C, \\
& \int_{\Omega}\left|e\left(u^{\varepsilon}(x)\right)\right|^{2} d x \leqslant C,
\end{aligned}
$$

i.e., there exists a non relabeled subsequence, such that $u^{\varepsilon} \rightarrow u^{0}$ in $V^{\varepsilon}$, and $u^{\varepsilon} \rightarrow u^{0}$ in $X$.

We now proceed to prove Theorem 1.

Proof of Theorem 1. We need to show that $\forall u_{0} \in X$

- step 1: $\exists u^{\varepsilon} \rightarrow u^{0}$ in $X$ such that $\lim \sup _{\varepsilon \rightarrow 0} F^{\varepsilon}\left(u^{\varepsilon}\right) \leqslant F^{0}\left(u^{0}\right)$;

- step 2: $\forall u^{\varepsilon} \rightarrow u^{0}$ in $X \liminf _{\varepsilon \rightarrow 0} F^{\varepsilon}\left(u^{\varepsilon}\right) \geqslant F^{0}\left(u^{0}\right)$.

To prove step 1, take the constant sequence $u^{\varepsilon}=u^{0}$ and go to the limit in $F^{\varepsilon}\left(u^{0}\right)$. To prove step 2 , let $k^{m}$ be a positive constant and introduce the following functional

$$
F^{\varepsilon, k^{m}}(v)=\left\{\begin{array}{l}
\int_{\Omega_{ \pm}^{\varepsilon}} a^{ \pm} e(v): e(v) d x+k^{m} \varepsilon \int_{B^{\varepsilon}} e(v): e(v) d x \quad \text { if } v \in V_{\varepsilon}, \\
+\infty \quad \text { if } v \in X \backslash V_{\varepsilon} .
\end{array}\right.
$$

We also take $u^{ \pm} \in C^{\infty}\left(\Omega^{ \pm}\right)$and introduce the regularizing sequence [34]

$$
\left(R^{\varepsilon} u\right)\left(\hat{x}, x_{3}\right)= \begin{cases}\frac{1}{2} S^{\varepsilon} u(\hat{x})+\frac{x_{3}}{\varepsilon}[u]_{\varepsilon}(\hat{x}) \quad \text { if }\left|x_{3}\right| \leqslant \frac{\varepsilon}{2} \\ u^{ \pm}\left(\hat{x}, x_{3}\right) & \text { if }\left|x_{3}\right|>\frac{\varepsilon}{2}\end{cases}
$$

with $S^{\varepsilon} u(\hat{x})=u^{+}\left(\hat{x}, \frac{\varepsilon}{2}\right)+u^{-}\left(\hat{x},-\frac{\varepsilon}{2}\right)$. This sequence shows the following property

$$
\lim _{\varepsilon \rightarrow 0}\left|e\left(R^{\varepsilon} u\right)-\frac{1}{\varepsilon}[u]_{\varepsilon} \otimes_{s} e_{3}\right|_{L^{2}\left(B^{\varepsilon}\right)}^{2}=0,
$$

where $\otimes_{S}$ denotes the symmetrical tensorial product. We introduce a smoothing sequence $u_{n}^{0} \in\left(C^{\infty}(\Omega)\right)^{3}$ converging strongly to $u^{0}$ in $W^{1,2}\left(\Omega^{+} \cup \Omega^{-}\right)$.

In view of the convexity of the functional $F^{\varepsilon, k^{m}}$, we can write:

$$
F^{\varepsilon, k^{m}}\left(u^{\varepsilon}\right) \geqslant F^{\varepsilon, k^{m}}\left(R^{\varepsilon} u_{n}^{0}\right)+\int_{\Omega_{ \pm}^{\varepsilon}} a^{ \pm} e\left(R^{\varepsilon} u_{n}^{0}\right): e\left(u^{\varepsilon}-R^{\varepsilon} u_{n}^{0}\right) d x+k^{m} \varepsilon \int_{B^{\varepsilon}} e\left(R^{\varepsilon} u_{n}^{0}\right): e\left(u^{\varepsilon}-R^{\varepsilon} u_{n}^{0}\right) d x .
$$

The second term on the right-hand side tends to zero because of the definition of the regularizing sequence (19) and because of the convergence properties of sequences $u^{\varepsilon}$ and $u_{n}^{0}$. Using (20), and [30]

$$
\left|u^{\varepsilon}\left(\hat{x}, \pm \frac{\varepsilon}{2}\right)-u^{0}\left(\hat{x}, 0^{ \pm}\right)\right|_{L^{2}(S)} \rightarrow 0,
$$


we also have

$$
\lim _{\varepsilon \rightarrow 0} \lim _{n \rightarrow \infty} \varepsilon \int_{B^{\varepsilon}}\left|e\left(R^{\varepsilon} u_{n}^{0}\right)\right|^{2} d x=\lim _{\varepsilon \rightarrow 0} \int_{S}\left|\left[u^{0}\right]_{\varepsilon} \otimes_{s} e_{3}\right|^{2} d s=\int_{S}\left|\left[u^{0}\right] \otimes_{s} e_{3}\right|^{2} d s .
$$

Again using (20) and the regularity of $u^{\varepsilon}$, we obtain

$$
\lim _{\varepsilon \rightarrow 0} \lim _{n \rightarrow \infty} \varepsilon \int_{B^{\varepsilon}} e\left(R^{\varepsilon} u_{n}^{0}\right) \cdot e\left(u^{\varepsilon}\right) d x=\lim _{\varepsilon \rightarrow 0} \lim _{n \rightarrow \infty} \int_{B^{\varepsilon}}\left(\left[u_{n}^{0}\right]_{\varepsilon} \otimes_{s} e_{3}\right) \cdot\left(u_{, 3}^{\varepsilon} \otimes_{s} e_{3}\right) d x=\int_{S}\left|\left[u^{0}\right] \otimes_{s} e_{3}\right|^{2} d s .
$$

Therefore, the third term on the right-hand side of (21) tends to zero. Consider

$$
F^{\varepsilon, k^{m}}\left(R^{\varepsilon} u_{n}^{0}\right)=\int_{\Omega_{ \pm}^{\varepsilon}} a^{ \pm} e\left(u_{n}^{0}\right): e\left(u_{n}^{0}\right) d x+k^{m} \varepsilon \int_{B^{\varepsilon}} e\left(R^{\varepsilon} u_{n}^{0}\right): e\left(R^{\varepsilon} u_{n}^{0}\right) d x
$$

The first term on the right-hand side converges to $F^{0}\left(u^{0}\right)$. For the second term on the right-hand side, we use (23) and substituting into (21) gives

$$
\liminf _{\varepsilon \rightarrow 0} F^{\varepsilon, k^{m}}\left(u^{\varepsilon}\right) \geqslant F^{0}\left(u^{0}\right)+k^{m} \int_{S}\left|\left[u^{0}\right] \otimes_{s} e_{3}\right|^{2} d s .
$$

The coercivity of the elasticity tensor ensures that $F^{\varepsilon}(u) \geqslant F^{\varepsilon, \eta^{m} \varepsilon^{-1}}(u)=F^{\varepsilon, k^{m}}(u)$ for all $u \in V^{\varepsilon}$, and $k^{m}>0$. Therefore,

$$
\liminf _{\varepsilon \rightarrow 0} F^{\varepsilon}\left(u^{\varepsilon}\right) \geqslant F^{0}\left(u^{0}\right)
$$

and this completes step 2.

Comment 1. The $\Gamma$-convergence of $J^{\varepsilon}(v)$ to $F^{0}(v)-l(v)$ follows from Theorem 1 and from the convergence of $l^{\varepsilon}(v)$ to $l(v)$ [30].

Comment 2. Using standard arguments, we obtain the following limit equilibrium problem

$$
\left(P_{0}\right)\left\{\begin{array}{l}
\text { Find }\left(u^{0}, \sigma^{0}\right) \text { such that : } \\
\sigma_{i j, j}^{0}=-\varphi_{i} \quad \text { in } \Omega_{0}, \\
\sigma_{i j}^{0}=a_{i j k h}^{ \pm} e_{k h}\left(u^{0}\right) \text { in } \Omega^{ \pm} \\
u^{0}=0 \quad \text { on } \Gamma_{0} \\
\sigma^{0} n=g \quad \text { on } \Gamma_{1} \\
{\left[u^{0}\right]=0, \quad\left[\sigma^{0} e_{3}\right]=0 .}
\end{array}\right.
$$

Note that this limit problem involves only the adherents. The adhesive has vanished geometrically (as was to be expected) as well as mechanically, contrary to what happens in other models $[15,19,24,30]$. We also observe that, since the displacement and the traction vectors on $S$, have to be continuous, a very thin interphase will behave like a perfect interface.

The case of a soft adhesive is known to give a spring-type interface model, which is governed by a linear relation between the stress vector and the displacement vector jump [4]. One might intuitively expect the perfect interface model to be the limit case of the spring-type interface model when the spring parameters become sufficiently large, and our results confirm this prediction. With adhesives which are stiffer than the adherents, the limit model may not be so straightforward. A complete study of this case is presented in [8], where it is assumed that the elastic coefficients of the adhesive, $a_{i j k l}^{m}$, scales like $\varepsilon^{-a}$, with $a>0$. It was established in the latter study that there exist various regimes, depending on $a$. In particular, the perfect interface is the limit of the thin adhesive model as long as $a$ is equal to less than one. Theorem 1 extends this result to the case $a=0$.

\section{Illustration in one dimension}

We take the example of a bar $A B$ divided into three parts, $A C, C D$ and $D B$ (see Fig. 2). The bar is fixed at point $A$, and a given displacement $\delta$ is imposed at point $B$. We have

$$
\begin{aligned}
& A C=L_{1}-\varepsilon, \quad C D=\varepsilon, \quad D B=L_{2}, \quad L=L_{1}+L_{2}, \\
& u(0)=0, \quad u(L)=\delta .
\end{aligned}
$$




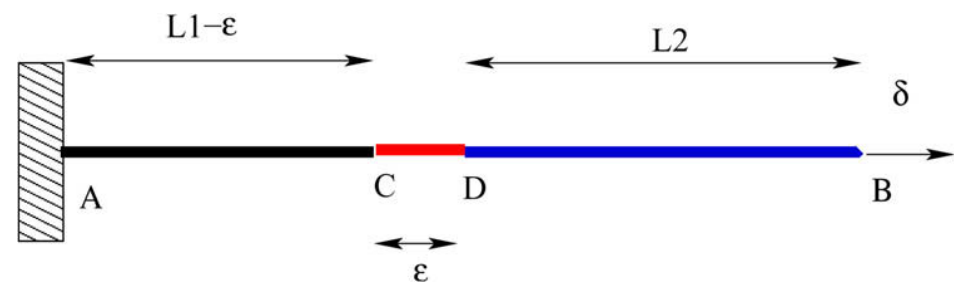

Fig. 2. Illustration: a simple bar under traction loading.

The elastic moduli of $A C, C D$ and $D B$ are denoted $E_{1}, E_{2}$ and $E_{3}$, respectively. At equilibrium, the displacement field is given by:

$$
\begin{aligned}
& u(x)=\frac{\sigma}{E_{1}} x \quad \text { if } 0 \leqslant x \leqslant L_{1}-\varepsilon, \\
& u(x)=\frac{\sigma}{E_{2}}\left(x-L_{1}+\varepsilon\right)+\frac{\sigma}{E_{1}}\left(L_{1}-\varepsilon\right) \quad \text { if } L_{1}-\varepsilon \leqslant x \leqslant L_{1}, \\
& u(x)=\frac{\sigma}{E_{3}}\left(x-L_{1}\right)+\frac{\sigma}{E_{2}} \varepsilon+\frac{\sigma}{E_{1}}\left(L_{1}-\varepsilon\right) \quad \text { if } L_{1} \leqslant x \leqslant L_{2} .
\end{aligned}
$$

The stress, which is constant, is given by $\sigma=\frac{\delta}{\frac{L_{2}}{E_{3}}+\frac{\varepsilon}{E_{2}}+\frac{1 L_{1}-\varepsilon}{E_{1}}}$
At order zero, we obtain:

$$
\begin{aligned}
& u^{0}(x)=\frac{\sigma^{0}}{E_{1}} x \quad \text { if } 0 \leqslant x \leqslant L_{1}, \\
& u^{0}(x)=\frac{\sigma^{0}}{E_{3}}\left(x-L_{1}\right)+\frac{\sigma}{E_{1}} L_{1} \quad \text { if } L_{1} \leqslant x \leqslant L_{2},
\end{aligned}
$$

where $\sigma^{0}=\frac{\delta}{\frac{L_{2}+\frac{L 1}{E_{1}}}{E_{1}}}$. In conclusion, we confirm that $\left[u^{0}\right]=\left[\sigma^{0}\right]=0$.

To check whether the solution at order zero provides a good approximation of the exact solution, the error $\left(u-u^{0}\right) / u$ in $(0, L)$ is given in Fig. 3 in an a-dimensionalized case.

The relative tension error can be easily computed:

$$
\frac{\sigma-\sigma^{0}}{\sigma^{0}}=\frac{\varepsilon}{L}\left(\frac{E_{1} / E_{2}-1}{E_{1} / E_{3}+1}\right) .
$$

Note that this error depends on the ratios between the elastic moduli of the materials. For example, consider the three cases of a lead joint with thickness $\varepsilon=0.01 \mathrm{~L}$ and rigidity $E_{2}=14 \mathrm{GPa}$ between a steel bar with $E_{1}=210 \mathrm{GPa}$ and a bar made of

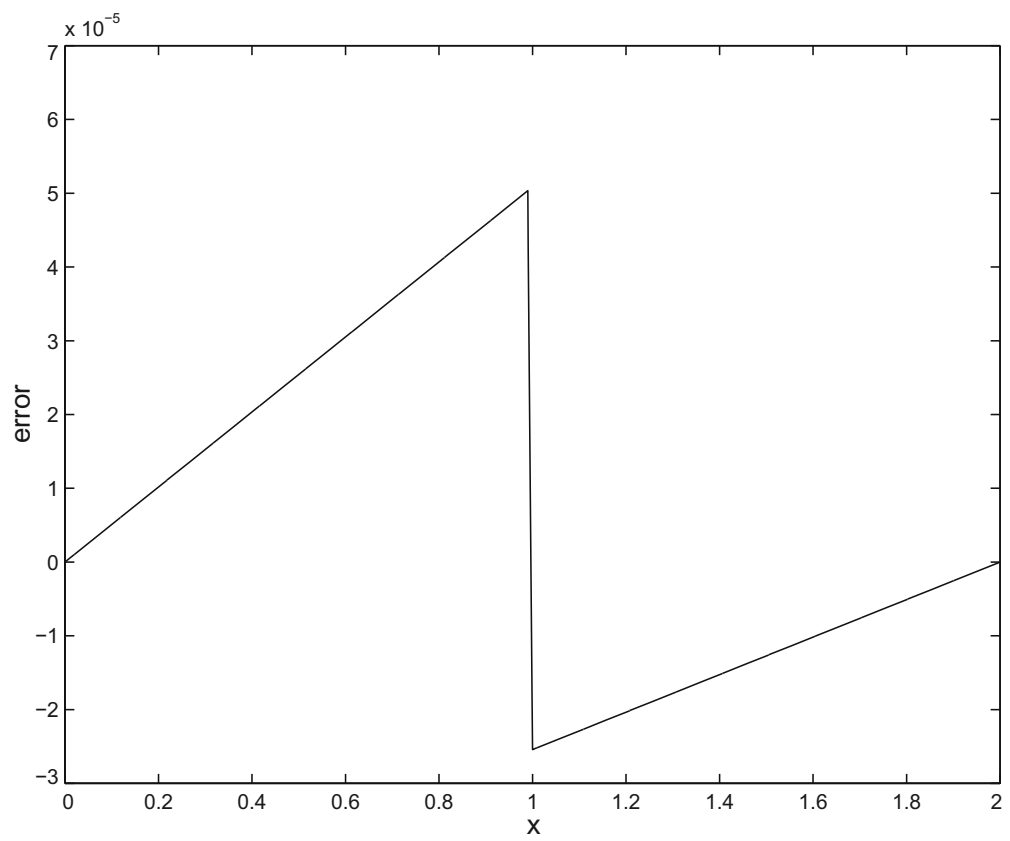

Fig. 3. Plot of the error $\left(u-u^{0}\right) / u$ in $(0, L)$ in an a-dimensionalized case $L_{1}=L_{2}=1, \varepsilon=0.01, E_{1}=1, E_{2}=1.5, E_{3}=2, \delta=1$. 
either steel $\left(E_{3}=210 \mathrm{GPa}\right)$, tungsten carbide $\left(E_{3}=640 \mathrm{GPa}\right)$ or aluminium $\left(E_{3}=70 \mathrm{GPa}\right)$. The error $(33)$ in these three cases is equal to $7 \%, 10.5 \%$ and $3.5 \%$, respectively. This simple example shows that in some cases, it can be necessary to improve the model, and it turns out that the most natural solution is to go to a higher order in the asymptotic analysis.

The situation is even more difficult in the three-dimensional case, because of the singularities which appear on the surfaces of the adhesive. If the adherents are made of the same material, then the singularities disappear at order zero and this gives a further reason for studying the equilibrium problem at a higher order. This was done in the following section.

\section{First order results}

In this section, the results obtained in $[1,24]$ are improved and generalized to the three-dimensional case, and we focus on the case of an isotropic adhesive with Lamé's coefficients $\lambda$ and $\mu$. We introduce the displacement field $u^{1}$ and the stress field $\sigma^{1}$ as the limits, as defined by the following Lemma, of suitable sub-sequences of $\frac{u^{\varepsilon}-u^{0}}{\varepsilon}$ and $\frac{\sigma^{\varepsilon}-\sigma^{0}}{\varepsilon}$, respectively. We take $D(A)$ to denote the space of the $C^{\infty}$ functions with compact support on the open set $A$ and $D^{\prime}(A)$ its dual space.

Lemma 3. Let $u^{\varepsilon}, u^{0}, \sigma^{\varepsilon}$ and $\sigma^{0}$ be the fields defined in Sections 2 and 3 , then there exist sub-sequences, non relabeled, such that

$$
\begin{aligned}
& \frac{u^{\varepsilon}-u^{0}}{\varepsilon} \rightarrow u^{1} \quad \text { in } L^{2}(\Omega) \text { (weak), } \\
& \frac{\sigma^{\varepsilon}-\sigma^{0}}{\varepsilon} \rightarrow \sigma^{1} \text { in } D^{\prime}(\Omega) \text { (weak). }
\end{aligned}
$$

This Lemma is proved in the Appendix. Contrary to the fields at order zero $u^{0}$ and $\sigma^{0} e_{3}$, which are continuous on the surface $S$, the fields $u^{1}$ and $\sigma^{1} e_{3}$ show discontinuities on $S$. The following Theorem, involving restrictions of the traction and displacement vectors $\sigma^{0} e_{3}$ and $u^{0}$ on $S$, relates their jumps.

\section{Theorem 2.}

$$
\begin{aligned}
& {\left[u_{\alpha}^{1}\right]=\frac{1}{\mu} \sigma_{\alpha 3}^{0}(\hat{x}, 0)-u_{3, \alpha}^{0}(\hat{x}, 0)-\frac{1}{2}\left(u_{\alpha, 3}^{0}\left(\hat{x}, 0^{+}\right)+u_{\alpha, 3}^{0}\left(\hat{x}, 0^{-}\right)\right), \quad \alpha=1,2,} \\
& {\left[u_{3}^{1}\right]=\frac{1}{\lambda+2 \mu} \sigma_{33}^{0}(\hat{x}, 0)-\frac{\lambda}{\lambda+2 \mu}\left(u_{1,1}^{0}(\hat{x}, 0)+u_{2,2}^{0}(\hat{x}, 0)\right)-\frac{1}{2}\left(u_{3,3}^{0}\left(\hat{x}, 0^{+}\right)+u_{3,3}^{0}\left(\hat{x}, 0^{-}\right)\right),} \\
& {\left[\sigma_{13}^{1}\right]=-\frac{4 \mu(\lambda+\mu)}{\lambda+2 \mu} u_{1,11}^{0}(\hat{x}, 0)-\mu u_{1,22}^{0}(\hat{x}, 0)-\frac{\mu(3 \lambda+2 \mu)}{\lambda+2 \mu} u_{2,21}^{0}-\frac{\lambda}{\lambda+2 \mu} \sigma_{33,1}^{0}(\hat{x}, 0)-\frac{1}{2}\left(\sigma_{13,3}^{0}\left(\hat{x}, 0^{+}\right)+\sigma_{13,3}^{0}\left(\hat{x}, 0^{-}\right)\right),} \\
& {\left[\sigma_{23}^{1}\right]=-\frac{4 \mu(\lambda+\mu)}{\lambda+2 \mu} u_{2,22}^{0}(\hat{x}, 0)-\mu u_{2,11}^{0}(\hat{x}, 0)-\frac{\mu(3 \lambda+2 \mu)}{\lambda+2 \mu} u_{1,12}^{0}(\hat{x}, 0)-\frac{\lambda}{\lambda+2 \mu} \sigma_{33,2}^{0}(\hat{x}, 0)-\frac{1}{2}\left(\sigma_{23,3}^{0}\left(\hat{x}, 0^{+}\right)+\sigma_{23,3}^{0}\left(\hat{x}, 0^{-}\right)\right),} \\
& {\left[\sigma_{33}^{1}\right]=-\sigma_{13,1}^{0}(\hat{x}, 0)-\sigma_{23,2}^{0}(\hat{x}, 0)-\frac{1}{2}\left(\sigma_{33,3}^{0}\left(\hat{x}, 0^{+}\right)+\sigma_{33,3}^{0}\left(\hat{x}, 0^{-}\right)\right),}
\end{aligned}
$$

in $D^{\prime}(S)$.

The proof of this Theorem will be given to Section 7 .

Comment 3. Using standard arguments, we obtain the equilibrium equations

$$
\left(P_{1}\right)\left\{\begin{array}{l}
\text { Find }\left(u^{1}, \sigma^{1}\right) \text { such that : } \\
\sigma_{i j, j}^{1}=0 \text { in } \Omega_{0}, \\
\sigma_{i j}^{1}=a_{i j k h}^{ \pm} e_{k h}\left(u^{1}\right) \text { in } \Omega^{ \pm}, \\
u^{1}=0 \text { on } \Gamma_{0}, \\
\sigma^{1} n=0 \text { on } \Gamma_{1}, \\
{\left[u^{1}\right] \text { given by }(35)_{1,2}, \quad\left[\sigma^{1} e_{3}\right] \text { given by }(35)_{3-5} .}
\end{array}\right.
$$

As with $\left(P_{0}\right)$, the limit problem $\left(P_{1}\right)$ involves only the adherents, since the adhesive has vanished geometrically. However, the adhesive has not disappeared from the mechanical point of view: it has been replaced by a mechanical constraint linking the jump in the displacement and traction vectors to the displacement and traction vectors at order zero and their derivatives. In particular, we note the presence of the tangential derivatives of $u^{0}$, showing non-local character of (35). Relations (35) suggest that at higher orders, a thin interphase behaves like an imperfect interface which has memorized the elastic behavior of the interphase.

Lastly, we note that (35) contain some extra terms in comparison with the analogous relations obtained in [1]. These terms are related to the normal derivatives of $u^{0}$ and $\sigma^{0} e_{3}$ in $x_{3}=0^{ \pm}$. 


\section{Numerical examples}

\subsection{Composite bar}

Let us take a bar composed of two identical adherents connected by an adhesive, which are subjected to symmetrical forces. Because of the symmetry, we will study only half of the bar, $A B$, which is fixed at one extremity, $A$, as shown in Fig. 4 .

Part $A B$ is composed of the adhesive $A C$ and the adherent $C B$, consisting of two different materials with elastic moduli $E_{1}$ and $E_{2}$, respectively. A force density $\phi(x)=\frac{b}{L-\varepsilon}(x-\varepsilon)+c$ is applied to $C B$. We have

$$
\begin{aligned}
& A C=\varepsilon, \quad C D=L-\varepsilon, \\
& u(0)=0, \quad E_{2} \frac{d u}{d x}(L)=0 .
\end{aligned}
$$

At equilibrium, the displacement field is given by:

$$
\begin{aligned}
& u(x)=(b / 2+c)(L-\varepsilon) \frac{x}{E_{1}} \quad \text { if } 0 \leqslant x \leqslant \varepsilon, \\
& u(x)=-\frac{b(x-\varepsilon)^{3}}{6(L-\varepsilon) E_{2}}-\frac{c(x-\varepsilon)^{2}}{2 E_{2}}+\frac{(b / 2+c)(L-\varepsilon)}{E_{2}}(x-\varepsilon)+(b / 2+c)(L-\varepsilon) \frac{x}{E_{1}} \quad \text { if } L-\varepsilon \leqslant x \leqslant L .
\end{aligned}
$$

The stress, which is not constant, is given by:

$$
\begin{aligned}
& \sigma(x)=(b / 2+c)(L-\varepsilon) \quad \text { if } 0 \leqslant x \leqslant \varepsilon, \\
& \sigma(x)=-\frac{b(x-\varepsilon)^{2}}{2(L-\varepsilon)}-c(x-\varepsilon)+(b / 2+c)(L-\varepsilon) \quad \text { if } L-\varepsilon \leqslant x \leqslant L .
\end{aligned}
$$

Note that the reaction at the fixed end is $R=(b / 2+c) L-(b / 2+c) \varepsilon$.

At order zero, we obtain:

$$
\begin{aligned}
& u^{0}(x)=-\frac{b x^{3}}{6 L E_{2}}-\frac{c x^{2}}{2 E_{2}}+\frac{(b / 2+c) L x}{E_{1}}, \\
& \sigma^{0}(x)=-\frac{b}{2 L} x^{2}-c x+(b / 2+c) L .
\end{aligned}
$$

It can easily be confirmed that $\left[u^{0}\right]=\left[\sigma^{0}\right]=0$. At order one, we obtain:

$$
\begin{aligned}
& u^{1}(x)=-\frac{b\left(x^{3} / L-3 x^{2}\right)}{6 L E_{2}}+\frac{c x}{E_{2}}-\frac{(b / 2+c)(L+x)}{E_{2}}+\frac{(b / 2+c) L}{E_{1}}, \\
& \sigma^{1}(x)=\frac{b\left(2 x-x^{2} / L\right)}{2 L}+b / 2 .
\end{aligned}
$$

It can be confirmed that $\left[u^{1}\right]=(b / 2+c) L\left(\frac{1}{E_{1}}-\frac{1}{E_{2}}\right)=\frac{1}{E_{1}} \sigma^{0}(0)-u_{, x}^{0}(0)$ and $\left[\sigma^{1}\right]=c=-\frac{1}{2}\left(\sigma_{, x}^{0}\left(0^{+}\right)+\sigma_{, x}^{0}\left(0^{-}\right)\right)$. The exact stress $\sigma$ and its approximation at order one $\sigma^{0}+\varepsilon \sigma^{1}$ can be seen in Fig. 5. The relative error here is less than $0.7 \%$.

\subsection{Simple shear loads in a composite block}

In this example, we take a composite solid consisting of three blocks with the same constant rectangular cross-section $S=\left(-l_{1}, l_{1}\right) \times\left(-l_{2}, l_{2}\right)$ and we set (Fig. 6)

$$
\begin{aligned}
& \Omega_{-}^{\varepsilon}=\left\{\left(x_{1}, x_{2}, x_{3}\right) \in \Omega: x_{3} \in\left(-h^{-},-\varepsilon / 2,\right)\right\}, \\
& B^{\varepsilon}=\left\{\left(x_{1}, x_{2}, x_{3}\right) \in \Omega: x_{3} \in(-\varepsilon / 2,-\varepsilon / 2)\right\}, \\
& \Omega_{+}^{\varepsilon}=\left\{\left(x_{1}, x_{2}, x_{3}\right) \in \Omega: x_{3} \in\left(\varepsilon / 2, h^{+}\right)\right\} .
\end{aligned}
$$

The base of the block is kept fixed

$$
u\left(\hat{x},-h^{-}\right)=0, \quad \hat{x} \in S .
$$

The base at $x_{3}=h^{+}$and the lateral surfaces at $x_{2}= \pm l_{2}$ are subjected to a given constant tangential load $\tau$

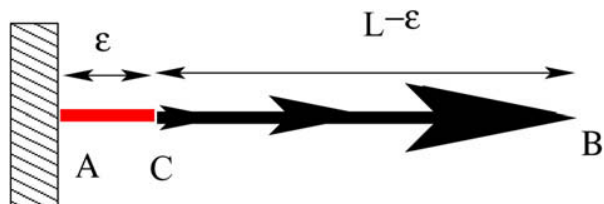

Fig. 4. A composite bar subjected to body forces. 


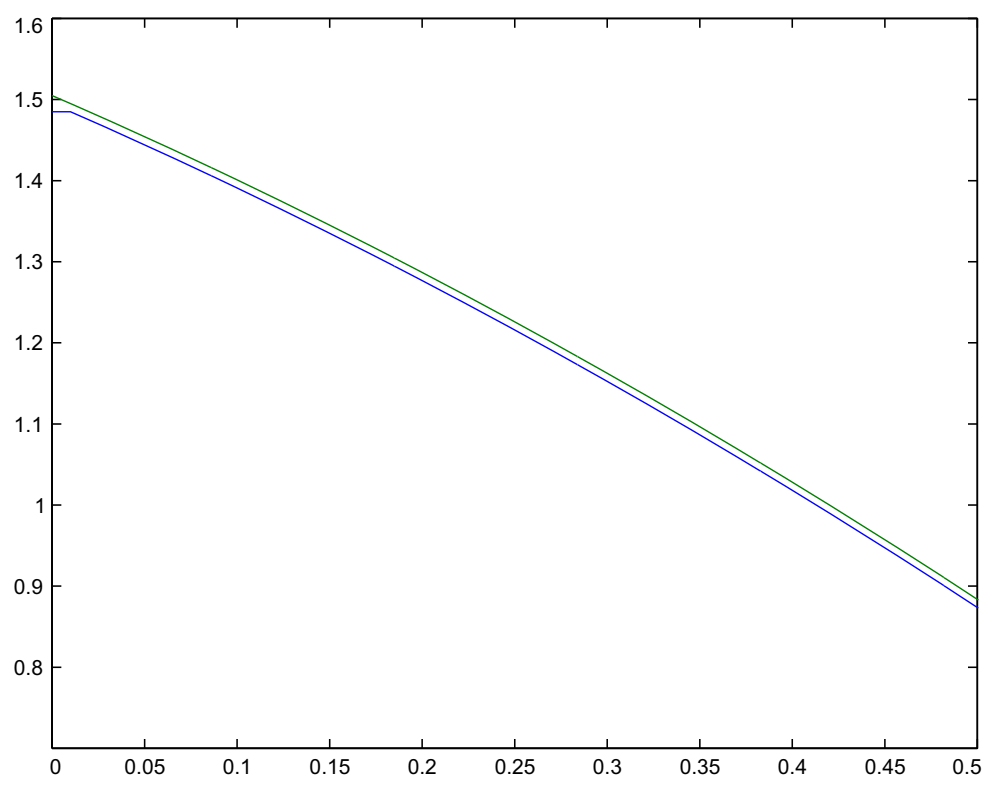

Fig. 5. Exact and order one stresses in an a-dimensionalized case $L=1, \varepsilon=0.01, b=c=1, E_{1}=89, E_{2}=210$.

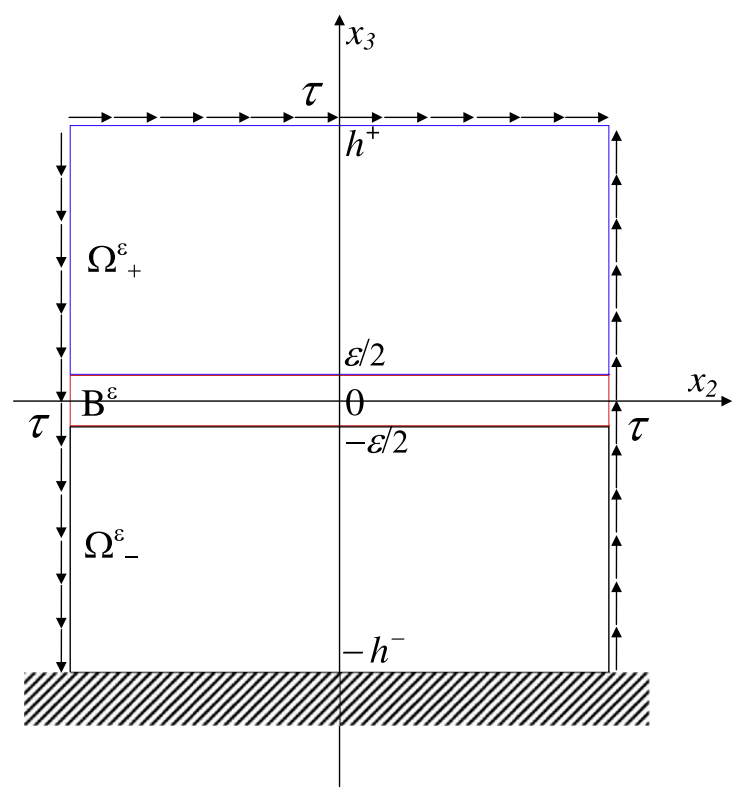

Fig. 6. Composite block subjected to tangential loading.

$$
\begin{aligned}
& \sigma^{\varepsilon}\left(\hat{x}, h^{+}\right) e_{3}=\tau e_{2}, \quad \hat{x} \in S, \\
& \sigma^{\varepsilon}\left(x_{1}, \pm l_{2}, x_{3}\right) e_{2}= \pm \tau e_{3}, \quad-l_{1} \leqslant x_{1} \leqslant l_{1}, \quad h^{-} \leqslant x_{3} \leqslant h^{+} .
\end{aligned}
$$

The adherents $\Omega_{ \pm}^{\varepsilon}$ are assumed to consist of isotropic, possibly different elastic materials and we take $\mu_{ \pm}, \lambda_{ \pm}$to denote their elastic constants.

The displacement solution to the equilibrium problem is the field

$$
u^{\varepsilon}=\left\{\begin{array}{l}
\left(\frac{\tau}{\mu_{-}}\left(x_{3}+h^{-}+\frac{\varepsilon}{2}\right)\right) e_{2} \quad \text { in } \Omega_{-}^{\varepsilon}, \\
\left(\frac{\tau}{\mu}\left(x_{3}+\frac{\varepsilon}{2}\right)+\frac{\tau}{\mu_{-}} h^{-}\right) e_{2} \text { in } B^{\varepsilon}, \\
\left(\frac{\tau}{\mu_{+}}\left(x_{3}-\frac{\varepsilon}{2}\right)+\frac{\tau}{\mu} \varepsilon+\frac{\tau}{\mu_{-}} h^{-}\right) e_{2} \text { in } \Omega_{+}^{\varepsilon},
\end{array}\right.
$$


corresponding to a piecewise homogeneous deformation obtained by applying a simple shear load to each block. We have

$$
\begin{aligned}
& u^{0}= \begin{cases}\left(\frac{\tau}{\mu_{-}}\left(x_{3}+h^{-}\right)\right) e_{2} & \text { in } \Omega_{-}, \\
\left(\frac{\tau}{\mu_{+}} x_{3}+\frac{\tau}{\mu_{-}} h^{-}\right) e_{2} & \text { in } \Omega_{+},\end{cases} \\
& u^{1}= \begin{cases}\frac{\tau}{2 \mu_{-}} e_{2} & \text { in } \Omega_{-}, \\
\left(-\frac{\tau}{2 \mu_{+}}+\frac{\tau}{\mu}\right) e_{2} & \text { in } \Omega_{+} .\end{cases}
\end{aligned}
$$

The limit $u^{1}$ is discontinuous at $x_{3}=0$ and

$$
\begin{aligned}
& {\left[u_{1}^{1}\right]=0,} \\
& {\left[u_{2}^{1}\right]=\frac{\tau}{\mu}-\frac{1}{2}\left(\frac{\tau}{\mu_{+}}+\frac{\tau}{\mu_{-}}\right),} \\
& {\left[u_{3}^{1}\right]=0,}
\end{aligned}
$$

these relations are in agreement with (35) $)_{1,2}$. Lastly, note that $\sigma^{\varepsilon}=\tau\left(e_{2} \otimes e_{3}+e_{3} \otimes e_{2}\right)=\sigma^{0}$. We therefore obtain $\sigma^{1}=0$, in line with relations $(35)_{3-5}$.

\section{Proof of Theorem 2}

In this section we give the proof of Theorem 2. For the sake of convenience, the proof is divided into two parts. The first part proves Eqs. (35) $)_{1,2}$ and the second one proves (35) $)_{3-5}$.

\subsection{Proof of the relation involving the displacement jump}

The proof is subdivided here into three steps. First, we link the jump in $u^{1}$ to a new variable denoted $\bar{u}^{1}$, which represents the "internal" jump mechanically in the thin layer.

\section{Lemma 4}

$$
\begin{aligned}
& \frac{\left[u^{\varepsilon}\right]_{\varepsilon}}{\varepsilon}-\bar{u}^{1}(\hat{x}) \quad \text { in } L^{2}(S) \text { (weak). } \\
& \frac{\left[u^{\varepsilon}-u^{0}\right]_{\varepsilon}}{\varepsilon}-\bar{u}^{1}(\hat{x})-\frac{1}{2} S u_{, 3}^{0}(\hat{x}, 0) \quad \text { in } L^{2}(S) \text { (weak), }
\end{aligned}
$$

where $\operatorname{Sv}(., 0)=v\left(., 0^{+}\right)+v\left(., 0^{-}\right)$

The proof of this Lemma is given in Appendix 3.

Comment 4. Since the displacement field $u^{1}$ is generally not regular enough for us to be able to define its traces on $S_{ \pm}$, the jump in $u^{1}$ across $S$ is defined via the limit given in Lemma 4. If the loads are sufficiently smooth, the trace of $u^{1}$ on $S$ will exist and the left hand side in (53) converges to the jump in $u^{1}$. Two examples, in which the equilibrium solution is sufficiently regular, have been presented in Section 6.

Comment 5. The result of Lemma 4 confirms the order one matching condition introduced into the matched asymptotic expansion (see for example [1, Eq. (5)]).

In the second step, the stresses resulting from the sequences $u^{\varepsilon}$ and $R^{\varepsilon} u^{\varepsilon}$ have to be introduced:

$$
\begin{aligned}
& \sigma_{m}^{\varepsilon}=a^{m} e\left(u^{\varepsilon}\right), \\
& \sigma_{ \pm}^{\varepsilon}=a^{ \pm} e\left(u^{\varepsilon}\right)=a^{ \pm} e\left(R^{\varepsilon} u^{\varepsilon}\right)=\bar{\sigma}_{ \pm}^{\varepsilon}, \\
& \bar{\sigma}_{m}^{\varepsilon}=a^{m} e\left(R^{\varepsilon} u^{\varepsilon}\right), \\
& \bar{\sigma}^{\varepsilon}= \begin{cases}\bar{\sigma}_{ \pm}^{\varepsilon} & \text { in } \Omega_{ \pm}^{\varepsilon}, \\
\bar{\sigma}_{m}^{\varepsilon} & \text { in } B_{\varepsilon},\end{cases}
\end{aligned}
$$

and the limit of $\int_{S} \bar{\sigma}_{m}^{\epsilon}\left(\hat{x}, x_{3}\right) \phi(\hat{x}) d \hat{x}$ can be shown to exist for any $\phi \in D(S)$. Denoting this limit $\int_{S} \bar{\sigma}^{0}(\hat{x}) \phi(\hat{x}) d \hat{x}$, we relate the weak limit $\bar{\sigma}^{0}$ to the weak limit $\bar{u}^{1}$.

\section{Proposition 1}

$$
\begin{aligned}
& \bar{\sigma}_{\alpha 3}^{0}=\mu\left(\bar{u}_{\alpha}^{1}+u_{3, \alpha}^{0}\right), \quad \alpha=1,2, \\
& \bar{\sigma}_{33}^{0}=\lambda\left(u_{1,1}^{0}+u_{2,2}^{0}\right)+(\lambda+2 \mu) \bar{u}_{3}^{1},
\end{aligned}
$$




$$
\begin{aligned}
& \bar{\sigma}_{\alpha \alpha}^{0}=\lambda\left(u_{1,1}^{0}+u_{2,2}^{0}+\bar{u}_{3}^{1}\right)+2 \mu u_{\alpha, \alpha}^{0}, \quad \alpha=1,2, \\
& \bar{\sigma}_{12}^{0}=\mu\left(u_{1,2}^{0}+u_{2,1}^{0}\right)
\end{aligned}
$$

in $D^{\prime}(S)$.

The proof of this Proposition is given in Appendix 3. To conclude the first part of the proof of Theorem 2, we give the following Lemma relating the values of $\bar{\sigma}^{0} e_{3}$ and $\bar{\sigma}^{0} e_{3}$.

\section{Lemma 5}

$$
\bar{\sigma}^{0} e_{3}=\sigma^{0} e_{3} \quad \text { a.e. on } S \text {. }
$$

This Lemma is proved in Appendix 3. The first two equations of Theorem 2 follow easily after collecting the results of Lemmas 4 and 5 and Proposition 1.

\subsection{Proof of the relation involving the traction vector jump}

Here the proof is subdivided into two steps. In the first step, the jump in $\sigma^{1} e_{3}$ related to a new variable denoted $\bar{\sigma}^{1} e_{3}$, which represents the "internal" jump in the traction vector in the thin layer mechanically.

\section{Lemma 6}

$$
\left(\frac{\left[\sigma^{\varepsilon}-\sigma^{0}\right]_{\varepsilon}}{\varepsilon}\right) e_{3} \rightarrow \bar{\sigma}^{1}(\hat{x}) e_{3}-\frac{1}{2} S \sigma_{, 3}^{0}(\hat{x}, 0) e_{3} \quad \text { in } D^{\prime}(S) \text { (weak) }
$$

The proof of this Lemma is identical to the proof of Lemma 4 given in Appendix 3.

Comment 6. The vector field $\bar{\sigma}^{1} e_{3}$ is generally not regular enough for us to be able to define its traces on $S_{ \pm}$, and we define therefore the jump in $\bar{\sigma}^{1} e_{3}$ across $S$ as done above in the case of the jump in $u^{1}$ in the Comment on Lemma 4.

In the second step, the vector field $\bar{\sigma}^{1} e_{3}$ is related to the stress tensor $\bar{\sigma}^{0}$. This is done in the following Proposition (which is proved in Appendix 4), based on the equilibrium equations related to the thin layer.

Proposition 2. Denoting $\bar{\sigma}_{\alpha 3}^{1}:=\mu \bar{u}_{\alpha, \alpha}^{1}, \alpha=1,2$, and $\bar{\sigma}_{33}^{1}:=\lambda\left(\bar{u}_{1,1}^{1}+\bar{u}_{2,2}^{1}\right)$, we have

$$
\bar{\sigma}_{i 3}^{1}=-\bar{\sigma}_{i 1,1}^{0}-\bar{\sigma}_{i 2,2}^{0}, \quad i=1,2,3
$$

in $D^{\prime}(S)$.

To complete the second step, the results of Propositions 1 and 2 and Lemma 5 are combined, giving:

$$
\begin{aligned}
& \bar{\sigma}_{13}^{1}=-\left(\frac{4 \mu(\lambda+\mu)}{\lambda+2 \mu} u_{1,11}^{0}+\mu u_{1,22}^{0}+\frac{\mu(3 \lambda+2 \mu)}{\lambda+2 \mu} u_{2,21}^{0}+\frac{\lambda}{\lambda+2 \mu} \sigma_{33,1}^{0}\right) \\
& \bar{\sigma}_{23}^{1}=-\left(\frac{4 \mu(\lambda+\mu)}{\lambda+2 \mu} u_{2,22}^{0}+\mu u_{2,11}^{0}+\frac{\mu(3 \lambda+2 \mu)}{\lambda+2 \mu} u_{1,12}^{0}+\frac{\lambda}{\lambda+2 \mu} \sigma_{33,2}^{0}\right) \\
& \bar{\sigma}_{33}^{1}=-\sigma_{31,1}^{0}-\sigma_{32,2}^{0}
\end{aligned}
$$

in $D^{\prime}(S)$.

Comment 7. Conditions (65) can be interpreted mechanically as follows: Let us assume that the adhesive is in a state of generalized plane stress, where the average out-of-plane stress vector, which coincides with the stress vectors $\sigma^{0} e_{3}$ [10], is given. Let us also assume that the body forces are nil.

Applying an averaging operation to the equilibrium equations across the adhesive, we obtain

$$
\begin{aligned}
& \bar{\sigma}_{11,1}+\bar{\sigma}_{12,2}+\left[\sigma_{13}\right]_{i}=0 \\
& \bar{\sigma}_{21,1}+\bar{\sigma}_{22,2}+\left[\sigma_{23}\right]_{i}=0 \\
& \bar{\sigma}_{31,1}+\bar{\sigma}_{32,2}+\left[\sigma_{33}\right]_{i}=0
\end{aligned}
$$

where the bar denotes the average. Taking the average constitutive equations of the adhesive and recalling that $\bar{\sigma}_{33}=\sigma_{33}^{0}$, we have

$$
\begin{aligned}
& \bar{e}_{33}=\frac{1}{\lambda+2 \mu} \sigma_{33}^{0}-\frac{\lambda}{\lambda+2 \mu}\left(\bar{e}_{11}+\bar{e}_{22}\right), \\
& \bar{\sigma}_{11}=\frac{4 \mu(\lambda+\mu)}{\lambda+2 \mu} \bar{e}_{11}+\frac{2 \mu \lambda}{\lambda+2 \mu} \bar{e}_{22}+\frac{\lambda}{\lambda+2 \mu} \sigma_{33}^{0},
\end{aligned}
$$




$$
\begin{aligned}
& \bar{\sigma}_{22}=\frac{2 \mu \lambda}{\lambda+2 \mu} \bar{e}_{11}+\frac{4 \mu(\lambda+\mu)}{\lambda+2 \mu} \bar{e}_{22}+\frac{\lambda}{\lambda+2 \mu} \sigma_{33}^{0}, \\
& \bar{\sigma}_{12}=2 \mu \bar{e}_{12} .
\end{aligned}
$$

Substituting into (66), recalling that $\bar{\sigma}_{\alpha 3}=\sigma_{\alpha 3}^{0}, \alpha=1,2$, and identifying the jumps $\left[\sigma_{i 3}\right], i=1,2,3$, with the jumps in components of $\bar{\sigma}^{1} e_{3}$, we obtain (65).

Note that Lemmas 4 and 3 introduce extra terms into the contact law (35). The jumps can be taken to result from superimposing the deformation of the adhesive, as described by (65) on the "matching" conditions (63 and 53 ) imposing continuous deformation between the adhesive and the adherents.

\section{Conclusion}

This study focuses on the asymptotic analysis of a joint consisting of two adherents and an adhesive, all of which having a similar rigidity. The aim of this paper was two-fold. First it was proposed to establish that the results obtained at order zero in [1] using matched asymptotic analysis can be exactly reproduced via $\Gamma$-convergence. Secondly, it was proposed to extend the results obtained at a higher order in [1] to the three-dimensional case, and this was accomplished by studying the properties of a (weakly converging) sequence of equilibrium solutions of the original three-dimensional problem.

The model obtained by studying the weak limit of this sequence, which is given by relations (65), gives a non-local model for the interface relating the jump in the stress vector to the jump in the displacement along the interface, along with their derivatives. It is worth noting that the relations (65) contain some extra terms in comparison with the corresponding relations obtained in [1]. These terms are seen to be necessary, as shown by the simple examples presented above.

This study should lead to many developments. It would be interesting to obtain an energy interpretation of (65) by performing the asymptotic $\Gamma$-expansion of the energy (13).

Another interesting extension would consist in taking the adhesive to be curved: an example of a composite cylindric assemblage given in [22] shows that in this case, the elastic constants in the relations involving imperfect interfaces are different from those occurring in (65).

In glue-bonding processes, the presence of residual stresses may determine the resistance of the joint, and it would be of interest to extend relations (65) to account for the residual stress. Lastly, implementing this model in a finite element code might make it possible to apply the imperfect interface model (65) to a whole range of practical engineering problems.

\section{Appendix 1. Proof of Lemma 2}

For the solution to $\left(\bar{P}_{\varepsilon}\right)$, we have

$$
\int_{\Omega^{\varepsilon}} a^{ \pm} e\left(u^{\varepsilon}\right): e\left(u^{\varepsilon}\right) d x+\int_{B^{\varepsilon}} a^{m} e\left(u^{\varepsilon}\right): e\left(u^{\varepsilon}\right) d x=l\left(u^{\varepsilon}\right)
$$

Using the definition of the linear form $l$, the Cauchy-Schwartz inequality, and $\varepsilon<1$, we obtain

$$
\left|l\left(u^{\varepsilon}\right)\right| \leqslant C\left\{\left(\int_{B^{\varepsilon}}\left|e\left(u^{\varepsilon}(x)\right)\right|^{2} d x\right)^{1 / 2}+\left(\int_{\Omega^{\varepsilon}}\left|e\left(u^{\varepsilon}(x)\right)\right|^{2} d x\right)^{1 / 2}\right\} .
$$

The coercivity of the elasticity tensors is then used to obtain:

$$
\eta\left\{\int_{B^{\varepsilon}}\left|e\left(u^{\varepsilon}(x)\right)\right|^{2} d x+\int_{\Omega^{\varepsilon}}\left|e\left(u^{\varepsilon}(x)\right)\right|^{2} d x\right\} \leqslant \int_{\Omega^{\varepsilon}} a^{ \pm} e\left(u^{\varepsilon}\right): e\left(u^{\varepsilon}\right) d x+\int_{B^{\varepsilon}} a^{m} e\left(u^{\varepsilon}\right): e\left(u^{\varepsilon}\right) d x
$$

This gives (16). To obtain (17), use (16) combined with Lemma 3.1. The weak convergence in $V^{\varepsilon}$ follows from the Trace Theorem, and the strong convergence in $X$ follows from the Rellich-Kondrachov Theorem.

\section{Appendix 2. Proof of Lemma 3}

From Theorem 1, it can be said that

$$
u^{\varepsilon} \rightarrow u^{0} \quad \text { in } L^{2}\left(\Omega_{0}\right) \text { (strong). }
$$

Using a result obtained by Licht and Michaille [30, Lemma 3.3] and the continuity of $u_{0}$ in problem $\left(P_{0}\right)$, we obtain

$$
\left[u^{\varepsilon}\right]_{\varepsilon} \rightarrow 0 \text { in } L^{2}(S) \text { (strong) }
$$


A subsequence, non relabeled, can therefore be extracted such that

$$
\begin{aligned}
& \frac{u^{\varepsilon}-u^{0}}{\varepsilon} \rightarrow u^{1} \quad \text { in } L^{2}\left(\Omega_{0}\right) \text { (weak). } \\
& \frac{\left[u^{\varepsilon}\right]_{\varepsilon}}{\varepsilon}-\bar{u}^{1} \quad \text { in } L^{2}(S) \text { (weak). }
\end{aligned}
$$

\section{Appendix 3. Proofs of Lemma 4, Proposition 1 and Lemma 5}

Proof of Lemma 4. The first part of the Lemma is obtained using the same argument as for Lemma 3. The proof of the second part follows using (52), and noting that by density, using a Taylor's expansion at point zero and the continuity of $u^{0}$ across $\mathrm{S}$,

$$
\frac{\left[u^{0}\right]_{\varepsilon}}{\varepsilon}-\frac{1}{2} S u_{, 3}^{0}(\hat{x}, 0) \text { in } L^{2}(S) \text { (weak). }
$$

Proof of Proposition 1. We write the constitutive relations as follows:

$$
\begin{aligned}
& \left(\bar{\sigma}_{m}^{\epsilon}\right)_{\alpha \alpha}=\lambda\left(\frac{1}{2} S^{\varepsilon} u_{1,1}^{\varepsilon}+\frac{1}{2} S^{\varepsilon} u_{2,2}^{\varepsilon}+\frac{x_{3}}{\varepsilon}\left[u_{1,1}^{\varepsilon}+u_{2,2}^{\varepsilon}\right]_{\varepsilon}+\frac{1}{\varepsilon}\left[u_{3}^{\varepsilon}\right]_{\varepsilon}\right)+2 \mu\left(\frac{x_{3}}{\varepsilon}\left[u_{\alpha, \alpha}^{\varepsilon}\right]_{\varepsilon}+\frac{1}{2} S^{\varepsilon} u_{\alpha, \alpha}^{\varepsilon}\right), \\
& \left(\bar{\sigma}_{m}^{\epsilon}\right)_{12}=\mu\left(\frac{x_{3}}{\varepsilon}\left[u_{1,2}^{\varepsilon}+u_{2,1}^{\varepsilon}\right]_{\varepsilon}+\frac{1}{2} S^{\varepsilon} u_{1,2}^{\varepsilon}++\frac{1}{2} S^{\varepsilon} u_{2,1}^{\varepsilon}\right) \\
& \left(\bar{\sigma}_{m}^{\epsilon}\right)_{\alpha 3}=\mu\left(\frac{1}{\varepsilon}\left[u_{\alpha}^{\varepsilon}\right]_{\varepsilon}+S^{\varepsilon} u_{3, \alpha}^{\varepsilon}+\frac{x_{3}}{\varepsilon}\left[u_{3, \alpha}^{\varepsilon}\right]_{\varepsilon}\right) \\
& \left(\bar{\sigma}_{m}^{\epsilon}\right)_{33}=\lambda\left(\frac{1}{2} S^{\varepsilon} u_{1,1}^{\varepsilon}+\frac{1}{2} S^{\varepsilon} u_{2,2}^{\varepsilon}+\frac{x_{3}}{\varepsilon}\left[u_{1,1}^{\varepsilon}+u_{2,2}^{\varepsilon}\right]_{\varepsilon}+\frac{1}{\varepsilon}\left[u_{3}^{\varepsilon}\right]_{\varepsilon}\right)+2 \mu \frac{1}{\varepsilon}\left[u_{3}^{\varepsilon}\right]_{\varepsilon},
\end{aligned}
$$

where the derivatives are taken in the distributional sense. For a function $\phi \in D(S)$, we have

$$
\begin{gathered}
\left|\int_{S} \frac{x_{3}}{\varepsilon}\left[u_{i, \alpha}^{\varepsilon}\right]_{\varepsilon} \phi d x\right| \leqslant\left|\int_{S}\left[u_{i, \alpha}^{\varepsilon}\right]_{\varepsilon} \phi d x\right|=\left|\int_{S}\left[u_{i}^{\varepsilon}\right]_{\varepsilon} \phi_{, \alpha} d x\right| \rightarrow\left|\int_{S}\left[u_{i}^{0}\right]_{\varepsilon} \phi_{, \alpha} d x\right|=0, \quad \alpha=1,2, \quad i=1,2,3, \\
\int_{S} \frac{1}{2} S^{\varepsilon} u_{i, \alpha}^{\varepsilon} \phi d x=-\int_{S} \frac{1}{2} S^{\varepsilon} u_{i}^{\varepsilon} \phi_{, \alpha} d x \rightarrow-\int_{S} u_{i}^{0} \phi_{, \alpha} d \hat{x}=\int_{S} u_{i, \alpha}^{0} \phi d \hat{x}, \quad \alpha=1,2, \quad i=1,2,3 .
\end{gathered}
$$

The result is obtained using (52).

Proof of Lemma 5. From the zero order result, we have (see Theorem 4.1 in [34])

$$
\sigma^{\varepsilon}-\sigma^{0} \text { in } L^{2}\left(\Omega_{0}\right) \text {. }
$$

From the continuity of the trace operator, we obtain

$$
\sigma_{ \pm}^{\varepsilon} e_{3} \rightarrow \sigma^{0} e_{3} \text { in } L^{2}(S)
$$

To prove the thesis in question, it is necessary to establish that the sequences $\sigma_{ \pm}^{\varepsilon} e_{3}$ and $\bar{\sigma}_{m}^{\epsilon} e_{3}$ have the same weak limit on $S$. Based on the continuity of the stress vector $\sigma^{\varepsilon} e_{3}$ on the surfaces $S_{ \pm}^{\varepsilon}$, we obtain

$$
0=\int_{S_{ \pm}^{\varepsilon}}\left(\sigma_{ \pm}^{\varepsilon} e_{3}-\sigma_{m}^{\varepsilon} e_{3}\right) \phi(\hat{x}) d \hat{x}=\int_{S_{ \pm}^{\varepsilon}}\left(\sigma_{ \pm}^{\varepsilon} e_{3}-\bar{\sigma}_{m}^{\varepsilon} e_{3}\right) \phi(\hat{x}) d \hat{x}+\int_{S_{ \pm}^{\varepsilon}}\left(\bar{\sigma}_{m}^{\varepsilon} e_{3}-\sigma_{m}^{\varepsilon} e_{3}\right) \phi(\hat{x}) d \hat{x} .
$$

Note that

$$
\begin{aligned}
& \left(\bar{\sigma}_{m}^{\varepsilon} e_{3}-\sigma_{m}^{\varepsilon} e_{3}\right)_{\alpha}=\mu\left(\frac{1}{\varepsilon}\left[u_{\alpha}^{\varepsilon}\right]_{\varepsilon}-u_{\alpha, 3}^{\varepsilon}\right) \quad \alpha=1,2, \\
& \left(\bar{\sigma}_{m}^{\varepsilon} e_{3}-\sigma_{m}^{\varepsilon} e_{3}\right)_{3}=(2 \mu+\lambda)\left(\frac{1}{\varepsilon}\left[u_{3}^{\varepsilon}\right]_{\varepsilon}-u_{3,3}^{\varepsilon}\right),
\end{aligned}
$$

on $S_{ \pm}^{\varepsilon}$. Using a density argument, we conclude that the restriction of $\frac{1}{\varepsilon}\left[u^{\varepsilon}\right]_{\varepsilon}-u_{3}^{\varepsilon}$ on $S_{ \pm}^{\varepsilon}$ converges to zero in $D^{\prime}(S)$, which, along with (81) and (82), means that the second term in (80) converges to zero. To complete the proof, we use (79) and recall that $\bar{\sigma}^{0}$ is the weak limit of the sequence $\bar{\sigma}_{m}^{\varepsilon}$ on $S$.

\section{Appendix 4. Proof of Proposition 2}

From (78), we deduce $\operatorname{div} \bar{\sigma}^{\varepsilon} \rightarrow 0$ in $D^{\prime}(\Omega)$ (weak). We take $\phi\left(\hat{x}, x_{3}\right)=\phi_{1}(\hat{x}) \phi_{2}\left(x_{3}\right), \phi_{2}\left(x_{3}\right)=1$ on $]-\frac{\varepsilon}{2}, \frac{\varepsilon}{2}[$ to obtain $\int_{S} \phi_{1}(\hat{x})\left(\int_{\frac{\varepsilon}{2}}^{\frac{\varepsilon}{2}} d i v \bar{\sigma}^{\varepsilon} d x_{3}\right) d \hat{x} \rightarrow 0$.

Therefore, up to a "rare" subsequence, $\int_{S} \phi_{1}(\hat{x})\left(\frac{1}{\varepsilon} \int_{\frac{\varepsilon}{2}}^{\frac{\varepsilon}{2}} d i v \bar{\sigma}^{\varepsilon} d x_{3}\right) d \hat{x} \rightarrow 0$. 


\section{Note that}

$$
\frac{1}{\varepsilon} \int_{-\frac{\varepsilon}{2}}^{\frac{\varepsilon}{2}} \bar{\sigma}_{i j}^{\varepsilon} d x_{3} \rightarrow \bar{\sigma}_{i j}^{0}, \in D^{\prime}(S),(\text { weak }), \quad i, j=1,2,3,
$$

and thus, after integrating by parts in the tangential derivatives,

$$
\frac{1}{\varepsilon} \int_{-\frac{\varepsilon}{2}}^{\frac{\varepsilon}{2}} \bar{\sigma}_{i \alpha, \alpha}^{\varepsilon} d x_{3} \rightarrow \bar{\sigma}_{i \alpha, \alpha}^{0}, \in D^{\prime}(S),(\text { weak }), \quad i=1,2,3, \alpha=1,2 .
$$

To complete the proof, note also that

$$
\frac{\left[\bar{\sigma}_{i 3}^{\varepsilon}\right]_{\varepsilon}}{\varepsilon} \rightarrow \bar{\sigma}_{i 3}^{1}, \in D^{\prime}(S),(\text { weak }), i=1,2,3
$$

\section{References}

[1] R. Abdelmoula, M. Coutris, J. Marigo, Comportement asymptotique d'une interphase élastique mince, Compte Rendu Académie des Sciences Série II 326 (1998) 237-242.

[2] E. Acerbi, G. Buttazzo, D. Percivale, Thin inclusions in linear elasticity: a variational approach, Journal fur die reine und Angewandte Mathematik 386 (1988) 99-115.

[3] E. Acerbi, G. Buttazzo, D. Percivale, A variational definition for the strain energy of an elastic string, Journal of Elasticity 25 (1991) 137-148.

[4] A. Ait-Moussa, Modélisation et étude des singularités d'un joint collé, Ph.D. Thesis, Université Montpellier II, 1989.

[5] G. Anzellotti, S. Baldo, D. Percivale, Dimension reduction in variational problems, asymptotic developments in $\gamma$-convergence, and thin structures in elasticity, Asymptotic Analysis 9 (1994) 61-100.

[6] H. Attouch, Variational Convergence for Functions and Operators, Pitman Advanced Publishing Program, 1984.

[7] Y. Benveniste, T. Miloh, Imperfect soft and stiff interfaces in two-dimensional elasticity, Mechanics of Materials 33 (2001) $309-323$.

[8] D. Caillerie, The effect of a thin inclusion of high rigidity in an elastic body, Mathematical Methods in Applied Sciences 2 (1980) 251-270.

[9] G. Dal-Maso, An Introduction to $\Gamma$-Convergence, Birkhauser, 1993.

[10] I. Doghri, Mechanics of Deformable Solids, Springer, 2000.

[11] H. Le Dret, A. Raoult, The nonlinear membrane model as variational limit of nonlinear three-dimensional elasticity, Journal de Mathématiques Pures et Appliquées 74 (1995) 549-578.

[12] W. Eckhaus, Asymptotic Analysis of Singular Perturbations, North-Holland, 1979.

[13] J.F. Ganghoffer, A. Brillard, J. Schultz, Modelling of the mechanical behaviour of joints bonded by a nonlinear incompressible elastic adhesive, European Journal of Mechanics A/Solids 16 (1997) 255-276.

[14] G. Geymonat, F. Krasucki, Analyse asymptotique du comportement en flexion de deux plaques collées, Compte Rendu Académie des Sciences Série I 325 (1997) 307-314.

[15] G. Geymonat, F. Krasucki, S. Lenci, Mathematical analysis of a bonded joint with a soft thin adhesive, Mathematics and Mechanics of Solids 16 (1999) 201-225.

[16] Z. Hashin, Thermoelastic properties of particulate composites with imperfect interfaces, Journal of Mechanics and Physics of Solids 39 (1991) 745-762.

[17] Z. Hashin, Extremum principles for elastic heterogeneous media with imperfect interfaces and their application to bounding of effective moduli, Journal of Mechanics and Physics of Solids 40 (1992) 767-781.

[18] Z. Hashin, Thin interphase/imperfect interface in elasticity with application to coated fiber composites, Journal of Mechanics and Physics of Solids 50 (2002) 2509-2537.

[19] A. Klarbring, Derivation of the adhesively bonded joints by the asymptotic expansion method, International Journal of Engineering Science 29 (1991) 493-512.

[20] F. Krasucki, A. Mnnch, Y. Ousset, Analyse asymptotique d'un assemblage collé en élasticité non linéaire, Compte Rendu Académie des Sciences Série IIb 329 (2001) 429-434.

[21] F. Lebon, A. Ould-Khaoua, C. Licht, Numerical study of soft adhesively bonded joints in finite elasticity, Computational Mechanics 21 (1997) 134-140.

[22] F. Lebon, R. Rizzoni, Asymptotic development for bonded composite elastic structures: $\gamma$-limit and analytical examples, in: Proceedings of the 2006 ICAMEM Conference, vol. cd-rom, 2006.

[23] F. Lebon, R. Rizzoni, Asymptotic study of soft thin layer: the non convex case, Mechanics of Advanced Materials and Structures 15 (2008) 12-20.

[24] F. Lebon, R. Rizzoni, S. Ronel, Analysis of non-linear soft thin interfaces, Computers and Structures 82 (2004) 1929-1938.

[25] F. Lebon, S. Ronel, Asymptotic studies of Mohr-Coulomb and Drucker-Prager soft thin layers, International Journal of Steel and Composite Structures 4 (2004) 133-148.

[26] F. Lebon, S. Ronel, First order numerical analysis of linear thin layers, ASME Journal of Applied Mechanics 74 (2007) $824-828$.

[27] N.K.S. Lee, Z.Q. Li, Investigation of glue-bonding process on assembly accuracy, International Journal of Advances in Manufacturing Technology 32 (2007) 336-349.

[28] C. Licht, Comportement asymptotique d'une bande dissipative mince de faible rigidité, Compte Rendu Académie des Sciences Série I 317 (1993) $429-$ 433.

[29] C. Licht, G. Michaille, Une modélisation du comportement d’un joint collé élastique, Compte Rendu Académie des Sciences Série I 322 (1996) 295-300.

[30] C. Licht, G. Michaille, A modeling of elastic adhesive bonded joints, Advances in Mathematical Sciences and Applications 7 (1997) $711-740$.

[31] N. Nguetseng, E. Sanchez-Palencia, Stress concentration for defects distributed near a surface, in: P. Ladevèze (Ed.), Local Effects in the Analysis of Structures, Elsevier, 1985, pp. 55-74.

[32] A. Ould-Khaoua, F. Lebon, C. Licht, G. Michaille, Thin layers in elasticity: a theoretical and numerical study, in: Proceedings of the 1996 ESDA Conference, vol. 4, ASME, 1996, pp. 171-178.

[33] J. Sanchez-Hubert, E. Sanchez-Palencia, Introduction aux méthodes asymptotiques et à l'homogénisation, Masson, 1992.

[34] P. Suquet, Discontinuities and plasticity, in: Nonsmooth Mechanics and Applications, Springer-Verlag, 1988, pp. $315-323$.

[35] F. Zaittouni, Modélisation théorique et numérique d'interfaces, Ph.D. Thesis, Université Montpellier II, 2000.

[36] F. Zaittouni, F. Lebon, C. Licht, Etude théorique et numérique du comportement d'un assemblage de plaques, Compte Rendu Académie des Sciences Série Mécanique 330 (2002) 359-364. 